\title{
Incidental Pancreatic Lipomas: A Case Series on 64 Slice MDCT done for Different Pathologies
}

\author{
A D Dwivedi ${ }^{1}$, A Srivastava ${ }^{1}$, R Shukla $^{1}$, Patwari $^{1}$ \\ ${ }^{1}$ Dept. of Radiodiagnosis and Imaging, Institute of Medical Sciences, Banaras Hindu \\ University, Varanasi, UP, India
}

\begin{abstract}
Pancreatic lipomas are rare benign lesions, with only 50 cases being documented in the current literature to the best of our knowledge. We describe four cases of intrapancreatic lipomas from a single institution, all incidentally found using multi detector computed tomography within a 6-month period. Computed tomography depicted lipomas as homogeneous, nonenhancing, well-marginated lesions with a density coefficient consistent with adipose tissue.
\end{abstract}

Keywords: Lipoma; pancreas; Benign mesenchymal tumor; MDCT

\section{Introduction}

Tumors of the pancreas are fairly common and are the fourth leading cause of all cancerous deaths ${ }^{1}$. Most pancreatic neoplasm's occur as epithelial ductal adenocarcinomas, and the remaining $1 \%$ to $2 \%$ of these tumors arises neoplasms may be benign (lymphangioma, lipoma, teratoma, fibroma, neurofibroma, schwannoma, hemangioma, hemangioendothelioma, leiomyoma,and desmoid tumor) or malignant (lymphoma, pancreatoblastoma, liposarcoma, fibrous histiocytoma, hemangiopericytoma, and neuroectodermal neoplasms) and are identified histopathologically ${ }^{3}$. Benign mesenchymal fatty neoplasms such as lipomas are commonly found throughout the digestive tract, particularly the colon. In the pancreas, however, lipomas are very rare. We describe four cases of intrapancreatic lipomas from a single institution, all incidentally found using multi detector

Correspondence to: Amitnandan D Dwivedi Department of Radio diagnosis \& Imaging Institute of Medical Sciences, Banaras Hindu University, Varanasi-221005P, India E-mail:amitnandan21@yahoo.com computed tomography (CT) done for different reasons. All patients were imaged with 64 slice MDCT scanner (GE Light Speed VCT ct99) with 3 to 5-mm slice reconstructions. When intravenous contrast was used, 40 to $60 \mathrm{~mL}$ of Iodixanol was infused depending on the original clinical indication for the examination.

\section{Case Reports}

Case 1

A 50-year-old male patient was advised contrast enhanced CT of abdomen who presented with bleeding and an irregular hard mass per rectum. In addition to IV contrast, oral and rectal contrast was also instilled. Irregular, eccentric heterogeneously enhancing wall thickening of left lateral rectal wall was noted with few enlarged perirectal lymph nodes. In addition, an angular $0.4 \times 0.8 \times 1.1 \mathrm{~cm}$, well defined lesion was noted in the pancreatic neck, completely surrounded by the parenchyma (Fig. 1). It exhibited a homogeneous, low attenuation appearance with an attenuation coefficient of $-101 \mathrm{HU}$, similar to that of fat. No central or peripheral enhancement seen. This was reported as incidental pancreatic 


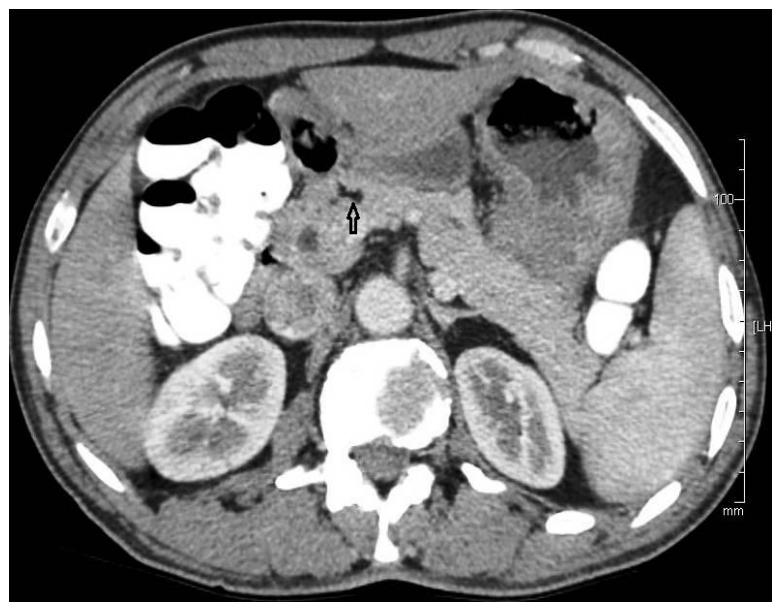

Figure 1: CT image in a patient with carcinoma rectum shows an incidental homogeneous lipoma in the pancreatic neck (arrow), with density matching subcutaneous and retroperitoneal adipose tissue.

lipoma on the basis of CT imaging in a case of carcinoma

rectum.

\section{Case 2}

A 60-year old male patient was advised contrast enhanced CT of thorax for evaluation of mediastinal enlargement as noted on chest $\mathrm{x}$-ray. Few enlarged, homogenous mediastinal lymph nodes were noted without any calcification or necrosis. Also noted was persistence of embryonic pattern of venous drainage with left brachiocephalic vein continuing as left SVC which in turn is draining into right atrium through coronary sinus. Incidentally, visualized sections of upper abdomen showed a rounded, sharply marginated, $1.1 \times 1.7 \times 1.8$ $\mathrm{cm}$, low-density lesion of fat attenuation was seen at neck-body junction of the pancreas (Fig. 2). It was homogeneous in appearance and completely encircled by pancreatic parenchyma, with an attenuation coefficient of $-96 \mathrm{HU}$ on the post contrast series. This had an appearance similar to that of previously described pancreatic lipomas.

\section{Case 3}

An 85-year old female patient presented to surgical OPD with a history of jaundice and vague abdominal pain. Examination revealed moderate icterus with a vague right hypochondriac mass. The patient was ordered contrast enhanced CT of abdomen. IV, oral and rectal contrast was used with CT acquisition of abdomen and pelvis obtained in $3-\mathrm{mm}$ sections.

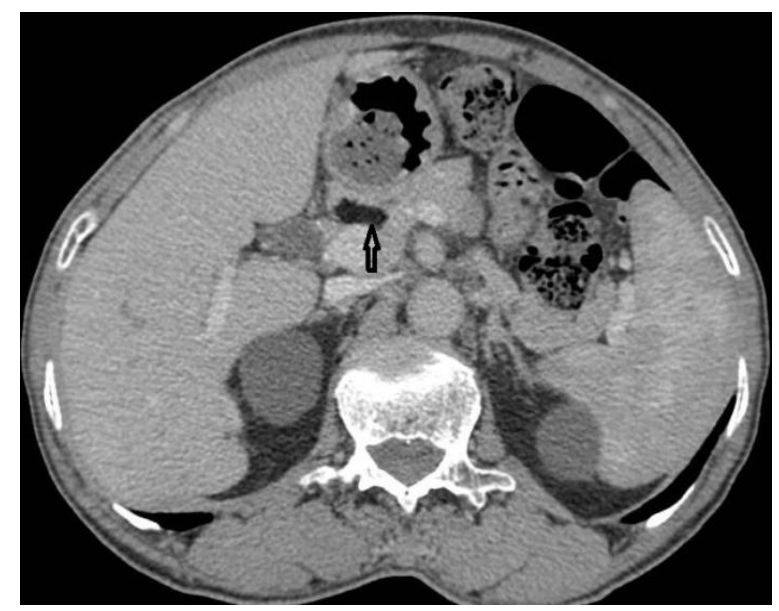

Figure 2: A 60-year old man with mediastinal lymphadenopathy and double SVC showed a well marginated fat-density lesion circumscribed in the neck body junction of the pancreas (arrow).

Irregularly thickened gall bladder wall with cholelithiasis and an ill defined minimally enhancing mass was noted infiltrating porta, segment IVb of liver, lateral duodenal wall and hepatic flexure of colon. Multiple enlarged periportal and peripancreatic lymph nodes were seen. CT features were suggestive of carcinoma gall bladder which was confirmed by FNAC. In addition a small, $0.6 \times 0.8 \times 0.7 \mathrm{~cm}$ oval lesion in the pancreatic neck was seen (Fig. 3). The attenuation coefficient for the lesion was consistent with fat, at $-87 \mathrm{HU}$, and was completely engulfed by pancreatic parenchyma. Findings were consistent with a pancreatic lipoma.

\section{Case 4}

An 80-year old male patient presented with history of high grade fever, chills and rigors and pain abdomen since 10 days. Contrast enhanced CT of abdomen was done which revealed hepatosplenomegaly with moderate ascites. Later the biochemical tests revealed 


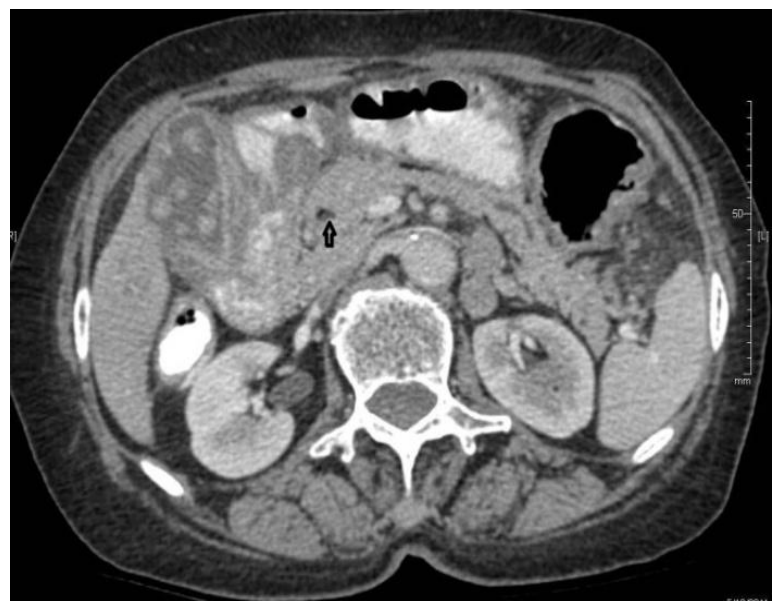

Figure 3: An 85-year old woman with carcinoma gall bladder. A small, oval, fatdensity lesion in the pancreatic neck (arrow) is completely engulfed by surrounding parenchyma.

typhoid antibodies. Incidentally identified was an elongated, $1.1 \times 1.2 \times 1.3 \mathrm{~cm}$ welldifferentiated, fat-density lesion in the neck of the pancreas (Fig. 4). This pancreatic lesion appeared well encapsulated with an attenuation of $-103 \mathrm{HU}$. This had the appearance of an intrapancreatic lipoma.

\section{Discussion}

Lipomas are benign tumors of homogeneous adipose tissue that is histopathologically identical to subcutaneous fat and entirely circumscribed by a thin $(<2 \mathrm{~mm})$ collagen capsule that may contain fibroreticular septa and scattered vessels ${ }^{2,4}$. The tumor is usually well defined on CT imaging and independent of the surrounding parenchyma, without infiltration of peripancreatic fat ${ }^{5}$. Lipomas are typically incidental findings in patients; however, several cases of lipoma have been reported in which the fatty tumor caused obstruction to the biliary or pancreatic duct or compression on the surrounding vessels, thereby requiring total surgical excision ${ }^{3,6-9}$. It should be noted that all lipomas that required surgical removal were located in the head of the pancreas. The size of a lipoma can vary greatly; the largest one reported by Boglino et al. ${ }^{7}$ measured $20 \times 30 \mathrm{~cm}$ and

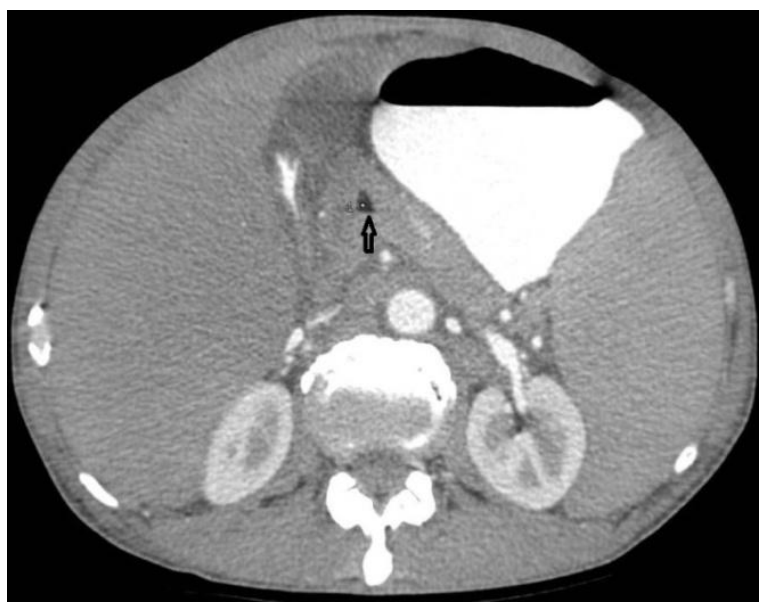

Figure 4: An 80-year old man with typhoid fever. Contrast-enhanced abdominal CT shows an elongated, benign-appearing fatty lesion in the pancreatic neck (arrow), which was interpreted as a lipoma.

required surgery, whereas others were smaller than $1.0 \mathrm{~cm}$.

Diagnostic imaging, in particular CT, can be readily used to diagnose lipomas. On CT, they appear as solid, homogeneous, wellmarginated, hypodense tumors with a density coefficient consistent with that of fat ${ }^{10}$. There is no contrast enhancement seen, with no islands of interposed parenchyma. Some investigators have asserted that a diagnosis can be made on CT alone ${ }^{3,8,11}$. With the distinguishing characteristics of lipoma, CT can differentiate this lesion from other types of localized pancreatic fatty masses, such as focal fatty infiltration (also known as fatty replacement, adipose atrophy, or lipomatosis) including lipomatous pseudohypertrophy, teratoma (mature dermoid cyst), and liposarcoma.

Our presented case series of pancreatic lipoma were incidental findings on CT imaging in patients who presented for different pathologies with no clinical sign and symptoms of pancreatic pathology. All cases were identified in a 6-month period and histopathologic confirmation was felt to be unnecessary in all cases due to the characteristic appearance and fat attenuation of the lesion. 


\section{Conclusion}

Pancreatic lipomas are very rare benign mesenchymal tumors, with only 50 cases found described in the medical literature. Lipomas of the pancreas are usually incidental finding. We have presented four intrapancreatic lipomas cases that were found incidentally at a single institution within a period of 6 months. Although pancreatic lipomas are described in the literature as rare entities, our findings of four cases within a short period seem to suggest otherwise. Steady advancement in diagnostic imaging tools that radiologists currently use may play a role in the increased reports of pancreatic lipoma. Histopathologic biopsy confirmation or invasive surgery is considered unnecessary for these benign areas of fat unless ductal or vessel obstruction is evident.

\section{References}

1. Kloppel G, Maillet B. Classification and staging of pancreatic nonendocrine tumors. Radiol Clin North Am i989; 27:105-119

2. Ferrozzi F, Zuccoli G, Bova D, et al. Mesenchymal tumors of the pancreas: CT findings. J Comput Assist Tomogr 2000; 24:622-627

3. Raut CP, Fernandez-del Castillo C. Giant lipoma of the pancreas: case report and review of lipomatous lesions of the pancreas. Pancreas 2003; 26:97-99
4. Kransdorf MJ, Bancroft LW, Peterson $\mathrm{JJ}$, et al. Imaging of fatty tumors: distinction of lipoma and welldifferentiated liposarcoma. Radiology 2002; 224:99-104

5. Itai Y, Saida Y, Kurosaki Y, et al. Focal fatty masses of the pancreas. Acta Radiol 1995; 36:178-181

6. Bigard MA, Boissel P, Regent D, et al. Intrapancreatic lipoma: first case in the literature. Gastroenterol Clin Biol 1989; 13:505-507

7. Boglino C, Inserra A, Silvano A, et al. Lipoma intrapancreatico: descrizione di un caso. Pediatr Med Chir 1993; 15:397-399

8. Di Maggio EM, Solcia M, Dore R, et al. Intrapancreatic lipoma: first case diagnosed with CT. AJR 1996; 167:56-57

9. Merli M, Fossati GS, Alessiani M, et al. A rare case of pancreatic lipoma. Hepatogastroenterology 1996; 43:734-736

10. Ryan MF, Hamilton PA, Smith AJ, et al. Radiologic features of pancreatic lipoma. Can Assoc Radiol J 2003; 54:41-44

11. Katz DS, Hines J, Math KR, et al. Using $\mathrm{CT}$ to reveal fat- containing abnormalities of the pancreas. AJR 1999;172:393-396 